\title{
A Unified Formulation of Nuclear Fluctuation Theory
}

\author{
M. BöHNING \\ Institut für Theoretische Physik der Universität Heidelberg, Heidelberg, Germany \\ (Z. Naturforschg. 21 a, 881-895 [1966] ; received 12 April 1966) \\ Dedicated to Professor Dr. W. Gentner on the occasion of his 60th birthday
}

\begin{abstract}
We here develop a formalism of nuclear fluctuation theory which we hope makes transparant the general structure of nuclear fluctuation theory and allows us to draw conclusions on the statistical properties of any kind of intensity which can be observed from a nuclear reaction. In the present paper the discussion concentrates on the case of compound reactions of purely fluctuating nature.
\end{abstract}

The statistical theory of fluctuations was initiated by ERICson ${ }^{1}$ in 1960 in the appendix of his review article on the statistical model of the compound nucleus. This was at a time when nuclear fluctuations had not yet been observed or recognized as such in scattering experiments. Ericson pointed out that the excitation functions and angular distributions of nuclear reactions involving a compound nucleus in its region of overlapping resonances should exhibit in high resolution experiments a specific kind of irregular fluctuations. As in the case of noise in electrical circuits or radio waves ${ }^{2}$ or other kinds of stochastic processes, one will consider these fluctuations as a single realization of an imaginary large ensemble of stochastic functions. One can then try to investigate, if not its details, so at least its statistical properties. Later ERICson ${ }^{3}$ gave a detailed account of the fluctuation theory by extending essentially the former theories of the average compound cross sections to the consideration of its variances and covariances. The assumption of many overlapping levels turned out to be very important in getting fairly simple results on these higher moments of the cross section.

The experimental investigations which were then started at several places, particularly in Tandem laboratories, to clarify this phenomenon of nuclear fluctuations could verify the predictions of ERICson. Their rather uniform structure and simple average behavior could be described by a few effective para-

1 T. Ericson, Adv. Phys. 9, 425 [1960] and Phys. Rev. Letters 5, 430 [1960].

2 T. ERIcson, CERN Preprint (1962) and Ann. Phys. N.Y. 23, 390 [1963].

3 C. E. Porter, Statistical Theories of Spectra: Fluctuations, Academic Press, New York and London 1965. meters, such as the average cross section, the mean direct contribution, the effective number of channels, the coherence energy and the coherence angle of the excitation functions and angular distributions, respectively.

ERICSON's theory and also the contributions of several other authors ${ }^{4-6}$ to the fluctuation theory were based upon the ideas of the statistical theory of isolated resonances and relied upon an extrapolation of its results to the region of overlapping resonances. It was the contribution of BRINK and STEPHEN ${ }^{4}$ that hinted at the importance of the central limit theorem of probability theory for the fluctuation theory. This theorem, if applied to the representation of the reaction amplitudes as a sum of many resonance amplitudes, each weighted with a BREIT WIGNER type resonance denominator, suggests that the reaction amplitudes in the fluctuation region should be Gaussian distributed random numbers.

Since this Gaussian behavior would show up under even weaker conditions than those assumed in the statistical resonance theory one can try to use it as a fundamental principle of the fluctuation theory without referring to other more specific assumptions. This principle has of course to be complemented by additional assumptions concerning the correlation of different amplitudes.

Treatments of the statistical theory along these lines, emphasizing its very simple and transparent statistical foundations have been given by STEPHEN ${ }^{4}$,

${ }^{4}$ D. M. Brink and R. O. Stephen, Phys. Letters 5, 77 [1963]. R. O. Stephen, Clarendon Lab. Report, Oxford 1963. D. M. Brink, R. O. Stephen, and N. W. Tanner, Nucl. Phys. 54, 577 [1964].

5 P. A. Moldauer, Phys. Letters 8, 70 [1964] and Phys. Rev. 135, B 642 [1964].

${ }^{6}$ K. F. Ratcliff, Technical Report, University of Pittsburg 1964. 
Ericson $^{7}$, Bondorf $^{8}$, and GibBs ${ }^{9}$. They lead to simple consequences, for example to the so called STEPHEN formula for the autocorrelation or fluctuation strength and to a class of RAYLEIGH type probability distributions of the cross section. But these simple results are unfortunately bound to the more restrictive and usually invalid assumption that the partial cross sections in different spin channels are uncorrelated and equal in strength.

If one tries to treat the fluctuation theory in a more realistic way one is immediately led to HAUSERFeshbach type expressions for the statistical expectation values of the various observable quantities. These expressions demand the execution of complicated kinematical summations, which hide the statistical implications behind trivial kinematics and besides that they rely upon a huge set of transmission coefficients as a measure of the relative or even absolute strength of the involved partial waves, which can be taken from presently known optical potentials not without some reservations. Furthermore there is the disadvantage that they can be discussed numerically only and this makes it rather difficult to furnish a clear idea of the full variety of manifestations the fluctuations can possibly exhibit.

Both methods are therefore not fully satisfying, not only in cases where one regards the fluctuations as a serious means of nuclear spectroscopy and one would like to know under which conditions discrepancies between experimental observations and theoretical predictions indicate a violation of the fundamental postulates of the theory or merely a wrong adjustment of some auxiliary parameters, but also in the investigation of other reaction mechanisms, where fluctuations can possibly occur as a disturbance and where one needs a precise estimate of these disturbing effects.

We will therefore try to give a formulation of the fluctuation theory which emphasizes its simple statistical structure and avoids the veiling of its immediate statistical consequences by the requirements of the kinematical summation, as well as the introduction of oversimplified assumptions. It will be easy to extend the consideration from the beginning to any observable intensity which can be measured in a nuclear reaction with two nuclei in the ingoing and

7 T. Ericson, Phys. Letters 4, 258 [1963].

8 J. P. Bondorf, in Nuclear Structure and Nuclear Reactions, Proceedings of the Ninth Summer Meeting of Nuclear Physicists, edited by N. Cindro (published by The Federal Nuclear Commission of Yugoslavia, 1964), p. 133. outgoing channels and to include in this way the predictions concerning arbitrary polarization experiments (chap. 1). A short review of the statistical postulates of the theory (chap. 2) will allow us to separate clearly the essential ones from those which can be regarded merely as specializations inferred from other physical reasonings or for the sake of convenience. The statistical information on an observable intensity will be expressed by the complete sequence of moments of its probability distribution, all moments being given in closed form (chap. 3 ) . The variety of all moment sequences which are admissible in the fluctuation theory is rather limited. This can be seen by introducing a sequence of generalized effective numbers of channels whose range can be shown to be restricted by several strong inequalities (chap. 4). From these results we will derive some general consequences concerning the possible variety of probability distributions which describe the statistical behavior of any fluctuating observable under arbitrary conditions.

For the sake of simplicity in the representation of these results, we will confine the final part of this investigation to reactions where no direct contributions occur. The discussion of the full problem, which can be treated with the same methods, will be given in a subsequent paper.

\section{Representation of Reaction Intensities}

The complete kinematical and dynamical information on a nuclear reaction of type

$$
\mathrm{A}+\mathrm{a} \rightarrow \mathrm{A}^{\prime}+\mathrm{a}^{\prime}
$$

(or shortly $\alpha \rightarrow \alpha^{\prime}$ ), relating directly to the properties of the individual nuclei in the asymptotic states is contained in the $M$-matrix. This matrix is essentially the $S$-matrix in the representation which is labelled by the characters and corresponding states in the free asymptotic wave functions, each forming a unitary representation of the kinematical symmetry group ${ }^{10}$. The characters can simply be identified with the conserved quantities mass, spin, and parity. The corresponding states are essentially the momenta and the spin orientations. The $M$-matrix

$$
\begin{aligned}
& M_{\mu^{\prime}, \nu^{\prime} ; \mu, \nu}(E ; \vartheta, \varphi)=\left\langle\begin{array}{ccc}
\alpha^{\prime} & I^{\prime} & i^{\prime} \\
\boldsymbol{p}^{\prime} & \mu^{\prime} & v^{\prime}
\end{array}|\mathrm{S}| \begin{array}{ccc}
\alpha & I & i \\
\boldsymbol{p} & \mu & \nu
\end{array}\right\rangle \quad(1.1) \\
& 9 \text { W. R. Gibss, Los Alamos Scientific Laboratory Report } \\
& \text { LA-3266 (1965). } \\
& 10 \text { H. Joos, Fortschr. Phys. 10, } 65 \text { [1962]. }
\end{aligned}
$$


is in this way is labelled by the spin orientations of the four particles involved. With respect to the spin indices the $M$-matrix is in general rectangular with $n=(2 I+1)(2 i+1)$ columns and $n^{\prime}=\left(2 I^{\prime}\right.$ $+1)\left(2 i^{\prime}+1\right)$ rows. We can assume the $M$-matrix to be reduced to the center of mass, where it suffices to specify the energy $E$ of relative motion and the differences $\vartheta$ and $\varphi$ in the directional angles of the initial and final relative momenta $\boldsymbol{p}$ and $\boldsymbol{p}^{\prime}$. In cases where we want to exhibit more clearly the symmetry of the $M$-matrix in its ingoing and outgoing indices we will specify the polar angles $\Theta, \Phi$ and $\Theta^{\prime}, \Phi^{\prime}$ of the momenta, considering them in analogy with the spin indices as continuously varying matrix indices of $M$.

The fundamental discrete symmetries, with respect to space reflection and inversion of motion, which one believes to hold in nuclear reactions, give rise to linear relations between the matrix elements and reduce the number of independent reaction amplitudes. We will denote this number by $N$. If the particles in either the initial or final state are identical nuclei, the distinction between $\mu$ and $\nu$ or $\mu^{\prime}$ and $v^{\prime}$ has no meaning. Yet we can keep the notation (1.1) by reducing the redundancy by an explicit statement of additional symmetry relations between the matrix elements.

The experimental setup of a certain reaction experiment can be described quantum mechanically by the density matrices of beam and target and by the efficiency matrix of the detectors which has properties similar to the density matrix and is essentially a shorthand notation for the orientation-sensitivity of subsequent scatterings or decays of the final products ${ }^{11}$.

In high resolution experiments, where energies, angles, and the kind of the particles are sharply distinguished, the density matrix $\varrho$ of the initial state is simply a KRONECKER product of two density matrices in the spin spaces of the particles A and a, if we use the same representation as that introduced for the $M$-matrix, and the efficiency matrix $\varepsilon$ is essentially the same in the spin spaces of the particles $\mathrm{A}^{\prime}$ and $\mathrm{a}^{\prime}$. Both matrices are hermitian and positive definite. If we assume the density matrix to be normalized by the condition $\operatorname{Tr}(\varrho)=1$ and the $M$-matrix so that the differential cross section of the reaction

11 K. Heht, Fortschr. Phys. 13, 557 [1965].

12 H. Faissner, Ergeb. Exakt. Naturw., Band 32, p. 180, Springer-Verlag, Berlin 1959. is given by

$$
\frac{\mathrm{d} \sigma}{\mathrm{d} \Omega}(E, \vartheta)=\operatorname{Tr}\left(M M^{+}\right)
$$

then all the other intensities which can be observed in this reaction can be represented by ${ }^{12}$

$$
\sigma(\varepsilon, \varrho)=\operatorname{Tr}\left(\varepsilon M \varrho M^{+}\right)
$$

(the notation $M^{+}$means the transposed complex conjugate of $M$ ), and are then given in the same units as the differential cross section. The cross section (1.2) appears as the special intensity $\sigma(1,1)$ where $\varrho$ and $\varepsilon$ are proportional to the unit matrix in the corresponding spin spaces.

The properties of $\varrho$ and $\varepsilon$ allow us to form the square root of these matrices and to write (1.3) in the form

$$
\sigma(\varepsilon, \varrho)=\operatorname{Tr}\left(\varepsilon^{1 / 2} M \varrho^{1 / 2} \varrho^{1 / 2} M^{+} \varepsilon^{1 / 2}\right) .
$$

After defining a generalized transition matrix $W(\varepsilon, \varrho)$ by

$$
W=\varepsilon^{1 / 2} M \varrho^{1 / 2}
$$

we find that the intensity

$$
\sigma(\varepsilon, \varrho)=\operatorname{Tr}\left(W W^{+}\right) \underset{\mu^{\prime}, \nu^{\prime}, \mu, \nu}{ }\left|W_{\mu^{\prime}, \nu^{\prime} ; \mu, \nu}\right|^{2}
$$

can be regarded as a sum of partial intensities and resembles in form completely the cross section as a sum of partial cross sections.

This is now essentially all we need from $S$-matrix theory, but it will help to simplify our further discussion considerably if we reformulate these expressions in terms of supermatrices and direct products of matrices ${ }^{13}$.

The last expression in (1.6) suggests the interpretation of the matrix $W$ as a supervector in a $\left(n \cdot n^{\prime}\right)$-dimensional unitary vector space, so that it is simply the square of this vector which gives the intensity $\sigma$. Since we had to use rectangular matrices in our context anyway, it is perhaps even better to dispense with the concept of a supervector and to interpret it as a one-column rectangular supermatrix. The multiplication of those matrices is the usual one, one has only to assert that the number of columns of the left factor is equal to the number of rows of the right factor. In this sense the product $W W^{+}$in (1.6) is a quadratic supermatrix

$$
\mathbf{W}=W W^{+} \text {. }
$$

13 E. P. Wigner, Group Theory, translated by J. J. Griffin, Academic Press, New York and London 1959. 
The indices of those supermatrices will be denoted by small Latin letters which serve as abbreviations of the 4-tuplets of original matrix indices:

$$
i=\left\{\mu^{\prime}, v^{\prime} ; \mu, v\right\} \text {. }
$$

In this notation the definition of $W$ in (1.5) can be interpreted as a product of a quadratic and a rectangular supermatrix

$$
W=\boldsymbol{H} M
$$

where the matrix $\boldsymbol{H}$, which depends upon $\varepsilon$ and $\varrho$, is defined by

$$
\begin{aligned}
\boldsymbol{H}_{i j}(\varepsilon, \varrho) & =\boldsymbol{H}_{\left\{\mu^{\prime}, v^{\prime} ; \mu, \nu\right\}\left\{\bar{\mu}^{\prime}, \nu^{\prime} ; \mu, \nu\right\}}(\varepsilon, \varrho) \\
& =\varepsilon_{\mu^{\prime}, v^{\prime} ; \bar{\mu}^{\prime}, \overline{v^{\prime}}}^{1 / 2} \times \varrho_{\mu, \bar{\nu} ; \mu, v}^{1 / 2} .
\end{aligned}
$$

The matrix $\boldsymbol{H}$ is what one calls a "direct product" of the matrices $\varepsilon^{1 / 2}$ and $\varrho^{1 / 2}$. It is easy to prove that $\boldsymbol{H}$ is hermitian and positive definite. If we introduce analogously to (1.7) the supermatrix

$$
\boldsymbol{M}=M M^{+}
$$

then the intensity $\sigma(\varepsilon, \varrho)$ is simply

$$
\begin{aligned}
\sigma(\varepsilon, \varrho) & =\operatorname{Tr}(\boldsymbol{W})=\operatorname{Tr}(\boldsymbol{H}(\varepsilon, \varrho) \boldsymbol{M} \mathbf{H}(\varepsilon, \varrho)) \\
& =\operatorname{Tr}\left(\boldsymbol{M} \mathbf{H}^{2}(\varepsilon, \varrho)\right) \\
& =\operatorname{Tr}\left(M^{+} \boldsymbol{H}^{2}(\varepsilon, \varrho) M\right)
\end{aligned}
$$

all forms being identical because the factors in a trace can be permutated cyclically.

In the sense of the last expression in (1.11) the intensity $\sigma$ is the quadratic form of the vector $M$ with the hermitian operator $\mathbf{H}^{2}(\varepsilon, \varrho)$. Our notation now clearly shows the resemblance to the theory of quantummechanical measurement, where a state is uniquely defined if its expectation values with respect to a complete set of commuting hermitian observables are known. In our case the $M$-matrix is determined by the full set of intensities $\sigma(\varepsilon, \varrho)$, their measurement meaning the execution of a "complete experiment" on the considered reaction.

There are other transformations which can be applied to the $M$-matrix. Particularly important are the recoupling transformations of the spin in the initial and final states, changes of the quantization axis of the spin, or rotations of the reference frame, which are all described by finite unitary matrices $U$ and $U^{\prime}$ in the ingoing and outgoing spin channels in the form

$$
\hat{M}=\left(U^{\prime}\right)^{+} M U .
$$

Forming the direct product $\boldsymbol{U}$ of $\left(U^{\prime}\right)^{+}$and $U^{+}$as in (1.9), which is again a unitary supermatrix, one can write (1.12) in our notation as

$$
\hat{M}=\boldsymbol{U} M
$$

and the corresponding transformation of $\boldsymbol{M}$ as

$$
\hat{\boldsymbol{M}}=\boldsymbol{U} \boldsymbol{M} \mathbf{U}^{+} .
$$

Now we can see immediately how those transformations affect the expressions for the intensities. We have

$$
\sigma(\varepsilon, \varrho)=\operatorname{Tr}(\boldsymbol{H} \boldsymbol{M} \boldsymbol{H})=\operatorname{Tr}(\hat{\boldsymbol{H}} \hat{\boldsymbol{M}} \hat{\boldsymbol{H}})
$$

where $\hat{\boldsymbol{H}}=\boldsymbol{U} \boldsymbol{H} \boldsymbol{U}^{+}$, but this is not equal to $\operatorname{Tr}(\hat{\boldsymbol{W}})$ except for the differential cross section, where $\boldsymbol{H}=\mathbf{1}$ and

$$
\frac{\mathrm{d} \sigma}{\mathrm{d} \Omega}=\sigma(1,1)=\operatorname{Tr}(\boldsymbol{M})=\operatorname{Tr}(\hat{\boldsymbol{M}}) .
$$

From (1.15) we see that the form of the expressions for the intensities does not actually depend upon the representation which we originally chose when we introduced the $M$-matrix in (1.1).

Besides these finite unitary transformations in the spin spaces, we will have to consider the infinite transformations which are connected with the decomposition of the $M$-matrix into partial waves. This decomposition plays an eminent rôle, because it allows us to study the consequences of the conservation of total angular momentum explicitly.

At the beginning of this chapter we had chosen as a representation the KRONECKER product of the asymptotic free particle states. The fundamental rotation invariance of nuclear systems implies that the $S$-matrix commutes with the unitary representations of the infinitesimal rotation generators. We therefore know that there must exist a canonical basis for the representation of the full kinematical group in which the representations of its elements and the $S$-matrix decompose simultaneously into irreducible representations.

The decomposition of the $M$-matrix into its irreducible components requires its matrix elements to be considered as functions of the directional angles of the relative momenta in their full range and therefore we write the $M$-matrix in the extended symmetrical form

$$
\widetilde{M}_{\mu^{\prime}, v^{\prime} ; \mu, v}\left(\Theta^{\prime}, \Phi^{\prime} ; \Theta, \Phi\right) .
$$

In the fully decomposed representation we have the $S$-matrix as a direct sum of irreducible submatrices which have to be labelled by the hitherto introduced 
characters and, in addition, by the total angular momentum $J$ and its orientation $M$, though the reduced $S$-matrix elements do not, in fact, depend on $M$.

Since in the decomposition sum the same irreducible representation can occur several times we need for further specification a degeneracy parameter which in the nonrelativistic case can be identified with the channel spins $s, s^{\prime}$ and the angular momenta $l, l^{\prime}$ contained in the ingoing and outgoing states. In the completely reduced form we will therefore label the $S$-matrix in the form

$$
S_{s^{\prime}, l^{\prime} ; s, l}^{J} \text {. }
$$

The unitary transformation $Z$ which effects the connection between the $S$-matrix and the $M$-matrix has the well known matrix elements

$Z_{i j}$

$=i^{l}\left(\begin{array}{cc|c}\boldsymbol{I} & i & s \\ \mu & \nu & \mu+\nu\end{array}\right)\left(\begin{array}{ccc}l & s & J \\ M-\mu-v & \mu+s & M\end{array}\right) Y_{l^{M-\mu-\nu}}(\Theta, \Phi)$.

Here the first matrix index is an abbreviation for $i=\{J, M, s, l\}$ and the other for $j=\{\mu, v, \Theta, \Phi\}$. A summation over $j$ is meant to contain an integration of the form

$$
\int_{0}^{2 \pi} \int_{0}^{\pi} \ldots \sin \Theta \mathrm{d} \Theta \mathrm{d} \Phi .
$$

We have written the usual Chebsch-Gordan-coefficients of the angular momenta so that the distinction between the characters in the upper line and the states in the lower becomes more obvious.

The transformations connecting the reduced $S$-matrix and the extended $M$-matrix can be written in the usual matrix notation as

$$
\begin{gathered}
\tilde{M}=\left(Z^{\prime}\right)^{+} S Z \\
S=Z^{\prime} \tilde{M} Z^{+} .
\end{gathered}
$$

and

The relation (1.19) is just what one calls the partial wave decomposition of the reaction amplitudes as long as the summation over the matrix indices, the kinematical summation, is not yet executed.

No difficulty arises in interpreting (1.20) and (1.21) in terms of supermatrices. If we introduce the direct product

$$
\mathbf{Z}=\left(Z^{\prime}\right)^{+} \times \boldsymbol{Z}
$$

as in (1.9) we can arrive at relations completely analogous to (1.12) to (1.16) by introducing a super-S-matrix

$$
\mathbf{S}=S S^{+}
$$

and the relation

$$
\begin{aligned}
\tilde{\boldsymbol{M}} & =\boldsymbol{Z} \boldsymbol{S} \mathbf{Z}^{+} \\
\mathbf{S} & =\mathbf{Z}^{+} \tilde{\boldsymbol{M}} \mathbf{Z}
\end{aligned}
$$

as analogues to (1.20) and (1.21). The supermatrix $\boldsymbol{Z}$ is again unitary.

If we confine the angles, which serve as matrix indices in $\boldsymbol{Z}$, to fixed values and do not allow to sum over them, then we can regard the matrix elements (1.19) only as defining a projection operator. The fixation of the angles acts like the application of two projection operators $P$ and $P^{\prime}$ to the extended $M$-matrix according to

$$
M=P^{\prime} \tilde{M} P \text {. }
$$

These projection operators are hermitian and idempotent, but they have no inverse. With the definition of a superprojector

$$
\boldsymbol{P}=P^{\prime} \times P
$$

we can get the relation

$$
\boldsymbol{M}=\boldsymbol{P} \tilde{\boldsymbol{M}} \boldsymbol{P}
$$

between the super- $M$-matrix and the extended super$M$-matrix. Now we see that the connection of $\boldsymbol{M}$ with the reduced $S$-matrix

$$
\boldsymbol{M}=\boldsymbol{P} \mathbf{Z ~ S ~ Z} \mathbf{Z}^{+} \boldsymbol{P}=\hat{\boldsymbol{P}} \mathbf{S} \hat{\boldsymbol{P}}
$$

is accomplished by an superprojector $\hat{\boldsymbol{P}}$ and can therefore not be inverted, as it was possible in (1.25).

Nevertheless it is possible to introduce (1.28) into the representation (1.11) of the intensities and to get the partial wave decomposition

$$
\begin{aligned}
\sigma(\varepsilon, \varrho) & =\operatorname{Tr}\left(\mathbf{S} \hat{\boldsymbol{P}} \boldsymbol{H}^{2}(\varepsilon, \varrho) \hat{\boldsymbol{P}}\right) \\
& =\operatorname{Tr}(\boldsymbol{S} \boldsymbol{A}(\varepsilon, \varrho ; \vartheta, \varphi)) .
\end{aligned}
$$

The matrix

$$
\boldsymbol{A}(\varepsilon, \varrho ; \vartheta, \varphi)=\hat{\boldsymbol{P}} \mathbf{H}^{2}(\varepsilon, \varrho) \hat{\boldsymbol{P}}
$$

with the specification of the directional angles now reduced again to the only essential scattering angles $\vartheta$ and $\varphi$, can be called the angular distribution supermatrix of the intensity $\sigma(\varepsilon, \varrho)$. It contains the whole information on the kinematics and on the experimental setup while $\boldsymbol{S}$ represents in a condensed form the dynamical peculiarities of the reaction.

For the introduction of the statistical hypotheses the formal expressions in (1.11) and (1.30) will serve as starting points. 


\section{The Statistical Hypotheses}

In this chapter we will formulate and discuss the basic assumptions of the fluctuation theory. We will not try to deduce them from more fundamental nuclear theories, but will rather take them as an axiomatic starting point. In order to give them the distinct character of a particular nuclear model, which one should be able to disprove or to improve in a definite way, we will bring the basic assumption into the form of a few hypotheses.

They are not intended to hold exactly under realistic conditions, but the resulting theory should mainly serve as a zeroth order approximation to the actual physical situation. Appropriate modifications of the basic assumptions could easily be incorporated into the theory at the price of less transparency. It is also for the purposes of these more extended theories that we intend to develop the formal structure of this idealized model in a well defined way.

The present model shall be applicable to heavy compound nuclei which are excited to high energies far above the thresholds for particle emission into many different fragmentation channels, where at the same time the density of resonant states, i. e. the number of different modes of internal motion, becomes exceedingly large.

The important parameter denoting this situation is the ratio of the mean total width $T$ of the compound states to their mean spacing $D$. This ratio $\Gamma / D$, which counts roughly the number of resonant amplitudes contributing at a given energy, should be so large that one can refer to the indications of the central limit theorem of statistics and say that the reaction amplitude as the sum of the many resonance amplitudes behaves with sufficient accuracy like a GAussian random number. The determination of a lower limit for $\Gamma / D$, which will guarantee this fluctuation condition to hold, requires further investigation.

The result will depend upon the peculiarities of the more or less irregular behavior of the individual resonance amplitudes, which is in practice rather unknown. The investigations of Moldauer ${ }^{5}$ which are based on the statistical theory of resonances, have shown that if one assumes the properties of isolated resonances to hold also in the range of overlapping levels, the value of $T / D$ need only be larger than one.
Idealizing this result and thereby dispensing with the particular assumptions under which it can be derived we will formulate the first and fundamental hypothesis of the fluctuation theory in the form:

I. In the range of strongly overlapping compound resonances the reaction amplitudes in the scattering matrix can be regarded as complex random numbers from a joint Gaussian distribution.

Since the character of a Gaussian distribution does not change under linear transformations of the random variables, the hypothesis I applies to the reaction amplitudes in any representation we want to use, because they are connected by unitary transformations, and it applies as well to the generalized amplitudes $W(\varepsilon, \varrho)$ defined in (1.5) .

We can therefore confine our further statistical investigations to these amplitudes $W$ since most of the results can very easily be extended to the other types of amplitudes on account of the relations given in chapter 1 .

There is only one point which should be mentioned in connection with the physical background of hypothesis I. If we relate this hypothesis to the elements of the reduced $S$-matrix (1.18) we must pay attention to the fact that because of the conservation of the total angular momentum $J$ which also serves as a specifying character on a class of submatrices of the reduced $S$-matrix, only those resonant states can contribute to the matrix elements which possess just this spin value $J$. The fluctuation condition must therefore hold for each class of resonances with given $J$ separately. Since the spin of a compound system cannot be arbitrarily large one must meet the situation where the density of high spin states has dropped down to such an extent that the fluctuation condition is no longer fulfilled there. Fortunately at the same time the contributions of these high spin resonances to the amplitudes $W$ of the observable intensities become very small because the associated partial waves with high angular momenta $l$ and $l^{\prime}$ are depressed by the centrifugal barrier. Therefore we will not worry about this effect.

The multivariate GAussian distribution, which is postulated in I for the reaction amplitudes $W$ in particular, can be specified completely by two sets of parameters, the vector of the mean values of the random variables and their correlation matrix. We denote the mean vector by

$$
d_{i}=\bar{W}_{i}
$$


and the remaining fluctuating amplitudes by

$$
f_{i}=W_{i}-d_{i} \text {. }
$$

The correlation matrix $\boldsymbol{C}$ is defined by its matrix elements

$$
\boldsymbol{C}_{i j}=\overline{f_{i} f_{j}^{*}} \text {. }
$$

It is a supermatrix with respect to the labels of the reaction amplitudes exactly in the sense of chapter 1 . One can see immediately from its definition that it is hermitian and non negative definite. The number of real parameters is $2 n n^{\prime}$ for the vector $d$ and $\left(n n^{\prime}\right)^{2}$ for the matrix $\boldsymbol{C}$.

The next assumption which we want to introduce concerns the random phase behavior of the reaction amplitudes. Stephen has shown ${ }^{4}$ that under the fluctuation condition the real and imaginary parts of a reduced $S$-matrix element have equal variances and vanishing covariance. This statement is essentially equivalent to saying that the phase $\alpha$ of a fluctuating $S$-matrix element is uniformly distributed over the whole angular range $0 \leqq \alpha<2 \pi$. This is exactly what one calls a random phase behavior. One can easily generalize this result to the covariances of the real and imaginary parts of two arbitrary $S$-matrix elements and obtains the generalized random phase relations

$\overline{\operatorname{Re}\left(S_{i}-\bar{S}_{i}\right) \operatorname{Re}\left(S_{j}-\overline{S_{j}}\right)}=\overline{\operatorname{Im}\left(S_{i}-S_{i}\right) \operatorname{Im}\left(S_{j}-\overline{S_{j}}\right)}$

$\overline{\operatorname{Re}\left(S_{i}-\bar{S}_{i}\right) \operatorname{Im}\left(S_{j}-S_{j}\right)}=-\overline{\operatorname{Im}\left(S_{i}-\bar{S}_{i}\right) \operatorname{Re}\left(S_{j}-\bar{S}_{j}\right) .}$

Random phase assumptions are usually introduced into the statistical theory of resonances as a basic postulate. We should emphasize here that under the fluctuation condition it is, to order $D / \Gamma$, a consequence of the fact that the many resonance amplitudes which contribute to the reaction amplitude are all weighted with BREIT-WIGNER denominators, which define poles in the lower half of the complex energy plane only, because of the causality of nuclear reactions.

The relations (2.4) are invariant under linear transformations of the random variables. Therefore we can formulate the second hypothesis in the form

II. The reaction amplitudes have a random phase distribution which is specified by the following relations between the covariances of the real and imaginary parts:

$$
\begin{aligned}
& \overline{\operatorname{Re}\left(f_{i}\right) \operatorname{Re}\left(f_{j}\right)}=\overline{\operatorname{Im}\left(f_{i}\right) \operatorname{Im}\left(f_{j}\right)} \\
& \overline{\operatorname{Re}\left(f_{i}\right) \operatorname{Im}\left(f_{j}\right)}=-\overline{\operatorname{Im}\left(f_{i}\right) \operatorname{Re}\left(f_{j}\right)} .
\end{aligned}
$$

The measure theoretical requirements of the probability theory demand that we treat the real and imaginary parts of complex random numbers as two separate real random variables. Such a treatment would destroy the simplicity of the supermatrix notation which we have introduced in chapter 1 and have used for the parametrization of the statistical assumption I in (2.1) to (2.3).

The relations (2.5) permit a pseudo-complex ${ }^{14}$ treatment which remains compatible with our former notation, and this is one of the reasons why we have emphasized the random phase hypothesis II. For this purpose we have to work with the characteristic function $X$ of the multivariate Gaussian distribution of the complex reaction amplitudes which we define by introducing complex associated variables $\Omega_{i}$ and $\Omega_{i}^{*}$ according to

$$
\begin{aligned}
X\left(\Omega_{1}, \Omega_{1}^{*}, \ldots\right) & =\overline{\exp \left\{i \sum_{i} \Omega_{j}^{*} f_{j}+\Omega_{j} f_{j}^{*}\right\}} \\
& =\exp \left\{-\frac{1}{4} \sum_{i j} C_{i j} \Omega_{i}^{*} \Omega_{j}\right\} .
\end{aligned}
$$

Further, the use of the characteristic function instead of the probability distribution as the basis of the statistical investigations is urged by the fact that it allows us to handle variables with singular correlation matrices without any difficulty, and we have to account for those cases whenever we have dependences between the random variables.

From the characteristic function $X$ all moments of the $f_{i}$ can be derived by differentiation at the origin according to the rule that application of a differentiation operator upon $X$ which contains the derivation $\partial / \partial \Omega_{i}^{*}$ resp. $\partial / \partial \Omega_{j}$ will result in the mean value of a product containing the factor $(i / 2) f_{i}$ resp. $(i / 2) f_{j}^{*}$. For example we have

$$
\left.\frac{\partial^{2} \chi}{\partial \Omega_{i}^{*} \partial \Omega_{j}}\right|_{0}=-\frac{1}{4} \overline{f_{i} f_{j}^{*}}=-\frac{1}{4} C_{i j} .
$$

Among the moments of the $f_{i}$ only those do not vanish that have an equal number of starred and unstarred factors. This is an immediate consequence of the random phase hypothesis II. The nonvanishing moments can be related to the matrix elements of $C$ according to the relation

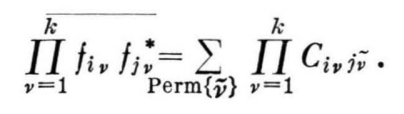

\footnotetext{
${ }^{14}$ L. A. Wainstein and V. D. Zubakov, Extraction of Signals from Noise, International Series in Applied Mathematics, translated by Silverman, Prentice Hall 1962.
} 
The sum is over all permutations of the numbers $1, \ldots, k$. As a simple example we quote

$$
f_{i} f_{k}^{*} f_{j} f_{l}^{*}=C_{i k} C_{j l}+C_{i l} C_{j k} .
$$

The use of the characteristic function as a moment generator would also be adventageous if the fundamental hypothesis $\mathrm{I}$ is violated to the order $D / T$. In this case additional higher terms in $\Omega$ and $\Omega^{*}$ of the order $D / T$ would occur in the exponent of the last expression in (2.7) with the so called higher cumulants appearing in the coefficients of these terms. These terms would then also produce additional terms of the order $D / \Gamma$ in the moments of the amplitudes $f_{i}$.

In order to make the fluctuation theory a model whose validity can be tested statistically and which allows us to estimate its parameters by comparison with observations, we must define how to obtain samples of sufficient size for statistical inference. At a fixed energy one can at best observe the $N$ amplitudes $M_{i}$ which are the elements of the $M$ matrix and the set of these amplitudes represents only one random event which does not permit any statistical inference. The size of the sample can only be extended, if we assume that the parameters, the mean vector $d$ and the correlation matrix $\boldsymbol{C}$ are independent of the energy so that we are allowed to take the amplitudes at different energies as random events of the same distribution. This requirement is the content of the third hypothesis:

III. The reaction amplitudes are stationary stochastic functions with respect to the energy as the argument.

As a consequence of this hypothesis we can interprete the mean vector $d$ as the vector of the direct amplitudes. This interpretation is justified since the reaction time of a scattering process, i. e. the time delay between a wave which propagates freely and another one which interacts with a target, is proportional to the logarithmic derivative of the $S$-matrix elements with respect to the energy. The mean amplitudes are therefore just those which suffer no time delay by the reaction.

From the standpoint of the pure fluctuation theory the interpretation of the mean vector as the vector of direct amplitudes means merely a certain parametrization of the observable intensities without any attempt to understand the reason for their occurrence. If one intends to specify the theory by introducing other physical arguments one would have to identify these direct amplitudes to some extent with the amplitudes of the direct reaction theories which in many cases turn out to be only slowly varying with energy.

The same two attitudes can be adopted with respect to the correlation matrix $\boldsymbol{C}$. One can on the one side consider it merely as a set of parameters which should be determined by experiments. It will be the essential object of our further considerations to get an insight into the problem as to what extent this aim can actually be achieved. On the other side, one refers to additional dynamical arguments which in this case are mostly based on BoHR's picture of the compound nucleus as a system which has such complicated modes of internal motion that it decays after a while without having any memory on how it was formed. The mathematical formulation of this picture leads essentially to a statement of complete absence of correlation between different amplitudes and to a factorization of the nonvanishing diagonal elements of the correlation matrix with respect to the in- and out-indices.

Since the statement that the hermitian correlation matrix is to be diagonal depends crucially on the choice of the representation for the amplitudes, this has to be specified. Because of the kinematically distinguished rôle which the reduced $S$-matrix plays among all the other possible representations one is inclined to relate the assumed "uncorrelatedness" to the amplitudes in this representation. Utilizing the unitarity of the total $S$-matrix which contains the transition amplitudes between all open channels, but neglecting the contribution of the direct amplitudes to the unitarity relation, one finds an expression for the diagonal elements of the correlation matrix in the reduced representation of the form

$$
\begin{aligned}
B_{i j}= & \overline{S_{i} S_{j}^{*}}=\overline{S_{l^{\prime}, s^{\prime} ; s l}^{J}\left(S_{l^{\prime}, \bar{s}^{\prime} ; \bar{l}}^{J}\right)^{*}} \\
& =\delta_{s \bar{s}} \delta_{l l} \delta_{\overline{s s^{\prime}}} \delta_{l^{\prime} l^{\prime}} \delta_{J J} \frac{T_{l^{\prime} s^{\prime}}^{J} T_{s}^{J}}{\sum_{c} T_{c}^{J}} .
\end{aligned}
$$

The $T_{l s}^{J}$ are the transmission coefficients in the indicated channels. They can in principle be derived from the elastic scattering amplitudes in these channels. The sum in the denominator, which expresses the restriction stemming from the unitarity, goes over all channels into which the part of the compound system having spin $J$ can decay. More refined results, which take into account the direct amplitudes properly, have been derived by Moldauer ${ }^{5}$. The introduction of the Borr-hypothesis (2.10) into the fluctuation 
theory via the expression (1.30) leads to HauserFeshbach expressions for the average behavior of the reaction intensities and will be seen to determine also the higher moments of the fluctuating amplitudes completely.

The correlation between reaction amplitudes at different energies $E_{1}$ and $E_{2}$ can be inferred from the same compound nucleus model, which assumes that the decay intensities emitted at different times are completely uncorrelated and drop down in the average exponentially with the mean life time $\hbar / \Gamma_{J}$ of the resonant states with spin $J$. Translating this statement by Fourier transformation into a statement on the energy correlation of the $S$-matrix elements one finds

$$
\overline{S_{i}\left(E_{1}\right) S_{j}^{*}\left(E_{2}\right)}=B_{i j} \frac{i \Gamma_{J}}{i \Gamma_{J}+\left(E_{1}-E_{2}\right)} .
$$

We have not listed the assumptions which lead to (2.10) and (2.11) among the basic hypotheses. This is because the parametrization which we will find appropriate to describe the statistical properties of the observable intensities does not depend in its formal structure on these particular relations. They are only important if one intends to determine the values of the parameters for a specific nuclear reaction in connection with other nuclear properties.

\section{The Statistical Moments of the Observable Intensities}

On the basis of the statistical assumptions which have been introduced in chapter 2 we will now discuss the statistical properties of the observable intensities $\sigma(\varepsilon, \varrho)$.

The complete statistical information on $\sigma$ as a random variable is contained in the distribution density $p(\sigma)$, but for reasons which have been mentioned in chapter 2 we will prefer to base our considerations on the characteristic function

$$
\chi(u)=\int_{0}^{\infty} e^{i u \sigma} p(\sigma) \mathrm{d} \sigma .
$$

The characteristic function $\chi(u)$ is an analytical function in a certain domain of the complex $u$-plane. The integral in (3.1) has only to be extended from 0 to $+\infty$ since $\sigma$ is non negative. Therefore we can also form the Laplace transform of $p(\sigma)$

$$
l(s)=\int_{0}^{\infty} e^{-s \sigma} p(\sigma) \mathrm{d} \sigma=\chi(i s) .
$$

From (3.1) and (3.2) one sees that one can get the moments of $\sigma$

$$
m_{r}(\sigma)=\int_{0}^{\infty} \sigma^{r} p(\sigma) \mathrm{d} \sigma
$$

simply by differentiation of $\chi(u)$ with respect to $(i u)$ and of $l(s)$ with respect to $(-s)$ at the origin in case that they exist. If the probability density $p(\sigma)$ goes to zero at least exponentially with increasing $\sigma$, a complete sequence of moments exists and this guarantees that $\chi(u)$ and $l(s)$ possess derivatives to any order at the origin. Except for pathological cases, the TAYLOR expansions of

$$
\chi(u)=1+\sum_{r=1}^{\infty} \frac{(i u)^{r}}{r !} m_{r}
$$

and of

$$
l(s)=1+\sum_{r=1}^{\infty} \frac{(-s)^{r}}{r !} m_{r}
$$

will have a non vanishing radius of convergence. If one knows the sequence of moments one can in principle get the probability distribution $p(\sigma)$ by analytical continuation of (3.4) and inversion of the Fourier transformation

$$
p(\sigma)=\frac{1}{2 \pi} \int_{-\infty}^{+\infty} e^{-i \sigma u} \chi(u) \mathrm{d} u
$$

and in a similar way from (3.5) by the inverse LAPLACE transformation. One can expand the logarithm of the characteristic function into a TAYLOR series and gets in this way a representation of $\chi$ in the form

$$
\chi(u)=\exp \left(\sum_{r=1}^{\infty} \frac{(i u)^{r}}{r !} \varkappa_{r}\right)
$$

The coefficients $\varkappa_{r} \mathrm{n}$ this series are the cumulants (or semi-invariants). The sequence of cumulants contains obviously as much information on the statistical distribution as the sequence of moments, but it expresses directly the deviation of a probability distribution from a Gaussian, which has only $x_{1}$ and $x_{2}$ as non vanishing cumulants. The first cumulant is equal to the mean value of $\sigma$, the second and third are equal to the second and third central moments

$$
\mu_{r}(\sigma)=\int_{0}^{\infty}\left(\sigma-m_{1}(\sigma)\right)^{r} p(\sigma) \mathrm{d} \sigma
$$

of $\sigma$, but the higher cumulants differ from the central moments of the same order. 
In the frame of the relations given above we will now discuss the statistical properties of $\sigma(\varepsilon, \varrho)$ which we have represented in (1.6) resp. (1.12) as the trace of the supermatrix $\boldsymbol{W}=W W^{+}$, defined in (1.7). The sequence of moments can immediately be derived from the statistical hypotheses of the fluctuation theory without any further assumptions or approximations.

Referring to the decomposition of $W$ in (2.1) and (2.2) into a direct supervector $d$ and a purely fluctuating supervector $f$ we can write $\boldsymbol{W}$ as the sum of three supermatrices

$$
\boldsymbol{W}=(d+f)(d+f)^{+}=\boldsymbol{D}+\boldsymbol{F}+\boldsymbol{I} .
$$

The supermatrix

$$
\boldsymbol{D}=d d^{+}
$$

represents the direct contribution, the supermatrix

$$
\boldsymbol{F}=f f^{+}
$$

the fluctuating contribution to the reaction amplitudes, and

$$
\mathbf{I}=d f^{+}+f d^{+}
$$

represents the interference terms between the direct and fluctuating parts. With these definitions the intensity $\sigma(\varepsilon, \varrho)$ is a sum of three traces

$$
\sigma(\varepsilon, \varrho)=\operatorname{Tr}(\boldsymbol{D})+\operatorname{Tr}(\boldsymbol{F})+\operatorname{Tr}(\mathbf{I}) .
$$

The mean value of $\sigma$ is on account of (2.3) directly given by

$$
m_{1}(\sigma)=\operatorname{Tr}(\boldsymbol{D})+\operatorname{Tr}(\boldsymbol{C}) .
$$

The higher moments (3.3) of $\sigma$ have to be calculated from the expression

$$
m_{r}(\sigma)=\overline{(\operatorname{Tr}(\boldsymbol{D})+\operatorname{Tr}(\boldsymbol{F})+\operatorname{Tr}(\boldsymbol{I}))^{r}} .
$$

This expression contains among many other terms the expectation value of the $r$-th power of $\operatorname{Tr}(\boldsymbol{F})$, which in case of vanishing direct amplitudes represents the observable intensity completely. In order to explain the structure of the full expression (3.14) at first in a simpler example, we will from now on assume that $\boldsymbol{D}=0$ and consequently $\boldsymbol{I}=0$ deferring the discussion of (3.14) in its general form to a subsequent paper. We hence assume that the moments of $\sigma$ are given by

$$
m_{r}(\sigma)=\overline{\operatorname{Tr}^{r}(\boldsymbol{F})}=\sum \underset{i_{1}, \ldots, i_{r}}{\sum} \overline{i_{i_{1}}, f_{i_{1}}^{*} \ldots f_{i_{r}} f_{i r}^{*}}
$$

In evaluating this mean value of any power of $\operatorname{Tr}(\boldsymbol{J})$ we can decompose the sum terms on the right side of (3.15a) according to (2.8) into sums of products of $r \boldsymbol{C}$-matrix elements. The first index of the $\boldsymbol{C}$-matrix elements appearing in the products runs from $i_{1}$ to $i_{r}$ while the second belongs to a permutation of the $i_{1}$ to $i_{r}$, the sum of products comprising all possible permutations. The result of the decomposition becomes more transparent if one resolves the permutations into conjugate classes with the same cycle structure. This is because the summation in (3.15a) over those indices belonging to one independent cycle gives rise to a trace of a power of the $\boldsymbol{C}$-matrix, the exponent being equal to the length of the cycle. If we characterize a cycle structure by the numbers $v_{1}$ of 1-cycles, $v_{2}$ of 2 -cycles, $\ldots, v_{r}$ of $r$-cycles, and write for it $\left[1^{\nu_{1}} \ldots r^{\nu_{r}}\right]$ or briefly $[v]$ then a term belonging to a permutation with cycle structure $[v]$ is equal to

$$
\prod_{\varrho=1}^{r} \operatorname{Tr}^{v} \varrho\left(\boldsymbol{C}^{\varrho}\right)
$$

Therefore writing $r[v]$ for the multiplicity of the cycle structure $[v]$ in the symmetric group of degree $r$, we have the result

$$
\overline{\operatorname{Tr}^{r}(\boldsymbol{F})}=\sum_{[v]} r[v] \prod_{\varrho=1}^{r} \operatorname{Tr}^{v} \varrho\left(\boldsymbol{C}^{\varrho}\right) .
$$

The multiplicities can be calculated from the formula

$$
r[\nu]=\frac{r !}{\underset{\varrho=1}{I I}\left(\varrho^{v} \varrho \cdot v_{\varrho} !\right)} .
$$

Concerning the central moments

$$
\mu_{r}(\sigma)=\overline{\operatorname{Tr}^{r}(\boldsymbol{F}-\boldsymbol{C})}
$$

one will find, either by looking at the structure of these quantities or by straight forward calculation, that they have a much simpler structure in so far as in

$$
\overline{\operatorname{Tr}^{r}(\boldsymbol{F}-\boldsymbol{C})}=\sum_{[v]} \delta_{0 v_{1}} \cdot r[v] \prod_{\varrho}^{r} \operatorname{Tr}^{v} \varrho\left(\boldsymbol{C}^{\varrho}\right)
$$

only those terms contribute whose cycle structure does not contain a 1-cycle. For illustration we quote the first few initial and central moments of the intensity $\sigma$ in Table 1 together with the corresponding cumulants. All moments can be expressed as homogeneous polynomials of traces formed from powers of $\boldsymbol{C}$, homogeneous in the sense that the sum of exponents of the traces and of the $\boldsymbol{C}$-matrices in the traces adds up to the order of the moments. This 


\begin{tabular}{|c|c|c|}
\hline Initial moments & Central moments & Cumulants \\
\hline $\begin{aligned} m_{1}(\sigma) & =\operatorname{Tr}(\boldsymbol{C}) \\
m_{2}(\sigma) & =\operatorname{Tr}^{2}(\boldsymbol{C})+\operatorname{Tr}\left(\boldsymbol{C}^{2}\right) \\
m_{3}(\sigma) & =\operatorname{Tr}^{3}(\boldsymbol{C})+3 \operatorname{Tr}(\boldsymbol{C}) \operatorname{Tr}\left(\boldsymbol{C}^{2}\right)+2 \operatorname{Tr}\left(\boldsymbol{C}^{3}\right) \\
m_{4}(\sigma) & =\operatorname{Tr}^{4}(\boldsymbol{C})+6 \operatorname{Tr}^{2}(\boldsymbol{C}) \operatorname{Tr}\left(\boldsymbol{C}^{2}\right)+ \\
& +3 \operatorname{Tr}^{2}\left(\boldsymbol{C}^{2}\right)+8 \operatorname{Tr}(\boldsymbol{C}) \operatorname{Tr}\left(\boldsymbol{C}^{3}\right)+6 \operatorname{Tr}\left(\boldsymbol{C}^{4}\right)\end{aligned}$ & $\begin{array}{l}\mu_{2}(\sigma)=\operatorname{Tr}\left(C^{2}\right) \\
\mu_{3}(\sigma)=2 \operatorname{Tr}\left(C^{3}\right) \\
\mu_{4}(\sigma)=3 \operatorname{Tr}^{2}\left(C^{2}\right)+6 \operatorname{Tr}\left(C^{4}\right)\end{array}$ & $\begin{array}{l}\varkappa_{1}(\sigma)=\operatorname{Tr}(C) \\
\varkappa_{2}(\sigma)=\operatorname{Tr}\left(C^{2}\right) \\
\varkappa_{3}(\sigma)=2 \operatorname{Tr}\left(C^{3}\right) \\
\varkappa_{4}(\sigma)=6 \operatorname{Tr}\left(C^{4}\right)\end{array}$ \\
\hline
\end{tabular}

Table 1. Moments and Cumulants of the distribution of $\sigma$.

homogeneity allows us the introduction of effective numbers $N_{k}$ of the rank $k$ by the relation

$$
N_{k}=\left[\frac{\operatorname{Tr}^{k}(\boldsymbol{C})}{\operatorname{Tr}\left(\boldsymbol{C}^{k}\right)}\right]^{1 /(k-1)} .
$$

With this definition one can represent the normalized initial moments of $\sigma$, which are identical with the moments of

in the form

$$
Q=\sigma / m_{1}(\sigma)
$$

$$
\frac{m_{r}(\sigma)}{m_{1}{ }^{r}(\sigma)}=m_{r}(\varrho)=1+\sum_{[v]} \frac{r[v]}{\prod_{\varrho=2}^{r} N_{\varrho}{ }^{v} \varrho} .
$$

A similar expression, corresponding to (3.19), holds for the normalized central moments. In an explicit form, the first few moments are given in Table 2.

Except for the index $k$, which specifies the rank of the effective numbers $N_{k}$, the moments (3.22) are identical in their form with the moments of a $\chi^{2}$-distribution of $2 N$ degrees of freedom. We will denote these moments by $g_{r}(N)$. They are given by the expression

$$
g_{r}(N)=\frac{\Gamma(N+r)}{N^{r} \Gamma(N)} .
$$

Here $\Gamma(z)$ denotes the Gamma-function.

It has been shown by several authors $4,7,9$ that under the assumption that the $M$-matrix contains $N$ independent and statistically uncorrelated amplitudes of equal strength the distribution density of a purely fluctuating cross section is simply a $\chi^{2}$-distribution of $2 \mathrm{~N}$ degrees of freedom of the form

$$
P_{N}(\varrho) \mathrm{d} \varrho=\frac{N}{\Gamma(N)}(N \varrho)^{N-1} e^{-N} \varrho \mathrm{d} \varrho .
$$

For later use, we will here quote its characteristic function

$$
\begin{aligned}
\chi_{N}(u) & =\left(1-\frac{i u}{N}\right)^{-N} \\
& =\exp \left(\sum_{r=1}^{\infty} \frac{(i u)^{r}}{r !} \frac{(r-1) !}{N^{r-1}}\right) .
\end{aligned}
$$

It is an analytical function in the whole $u$-plane with a pole of multiplicity $N$ at $u=-i N$. Comparing the last expression in (3.25) with (3.7) we can immediately find the cumulants of the distribution (3.24). They are very similar to the cumulants of the corresponding moments (3.22) of the general intensities which are given by

$$
\varkappa_{r}(\varrho)=\frac{(r-1) !}{N_{r} r-1} .
$$

From this relation and also from (3.22) compared with (3.23) we can learn that the only difference which occurs, if we extend the consideration from the cross section to an arbitrary reaction intensity and renounce the assumption of vanishing correlation and equal strenght, manifests itself in the dependence of the channel number $N$ on the rank $k$. We will therefore call the $N_{k}$ of (3.20) the "effective channel numbers of rank $k$ ".

\begin{tabular}{|l|l|c|}
\hline \multicolumn{1}{|c|}{ Initial moments } & Central moments & Cumulants \\
\hline$m_{1}(\varrho)=1$ & & $\varkappa_{1}(\varrho)=1$ \\
$m_{2}(\varrho)=1+\frac{1}{N_{2}}$ & $\mu_{2}(\varrho)=\frac{1}{N_{2}}$ & $\varkappa_{2}(\varrho)=\frac{1}{N_{2}}$ \\
$m_{3}(\varrho)=1+\frac{3}{N_{2}}+\frac{2}{N_{3^{2}}}$ & $\mu_{3}(\varrho)=\frac{2}{N_{3}^{2}}$ & $\varkappa_{3}(\varrho)=\frac{2}{N_{3}{ }^{2}}$ \\
$m_{4}(\varrho)=1+\frac{6}{N_{2}}+\frac{3}{N_{2}{ }^{2}}+\frac{8}{N_{3}{ }^{2}}+\frac{6}{N_{4}{ }^{3}}$ & $\mu_{4}(\varrho)=\frac{3}{N_{2}{ }^{2}}+\frac{6}{N_{4}{ }^{3}}$ & $\varkappa_{4}(\varrho)=\frac{6}{N_{4}{ }^{3}}$ \\
\hline
\end{tabular}

Table 2. Moments and Cumulants of the distribution of $\varrho$. 
The aim of our further considerations will be to show that under arbitrary conditions the effective channel numbers $N_{k}$ can differ only slightly from one insert one of them, preferably $N_{2}$, into (3.22) to get the moments of $\sigma$ with satisfying accuracy.

One should notice that the usual effective channel number $N_{\text {eff }}$, which is defined as the inverse of the Hauser-Feshbach expression for the normalized autocorrelation function, is exactly the same quantity as our $N_{2}$ referring to $\sigma(1,1)$ under the assumption (2.10).

\section{Properties of the Effective Channel Numbers}

Since the correlation matrix is hermitian, one can find a unitary matrix $\boldsymbol{V}$ which diagonalizes it into a matrix

$$
\tilde{\boldsymbol{C}}=\boldsymbol{V} \boldsymbol{C} \boldsymbol{V}^{+}
$$

whose matrix elements shall be denoted by

$$
\tilde{C}_{i j}=\lambda_{i} \delta_{i j}, \quad \lambda_{i} \geqq 0 .
$$

We observe that the diagonalizing matrix $\boldsymbol{V}$ can simultaneously be applied to the powers of $\boldsymbol{C}$ in all effective channel numbers $N_{\varrho}$ appearing in the moments (3.22) to bring them into the form

$$
N_{k}=\left[\left(\sum_{i=1}^{N} \lambda_{i}\right)^{k} / \sum_{i=1}^{N} \lambda_{i}{ }^{k}\right]^{1 /(k-1)} .
$$

Among the $\left(n \cdot n^{\prime}\right)$ eigenvalues of $\boldsymbol{C}$ at the most $N$ (the number of kinematically independent amplitudes) will not vanish. The transformation of $\boldsymbol{C}$ into its diagonal form and the fact that the moments of the intensity are invariant with respect to this transformation show that it is not the full set of $\left(n \cdot n^{\prime}\right)^{2}$ parameters of the $\boldsymbol{C}$-matrix, but only the $N$ nonvanishing eigenvalues which determine the statistical properties of $\sigma$.

This result holds for any particular intensity separately. The next question must be how the statistical properties of intensities observed in different experimental setups are related. It can easily be answered with the help of the expression (1.11), if we now specify the various matrices of the particular experiment explicitly by the corresponding value of $\varepsilon$ and $\varrho$. The correlation matrix $\boldsymbol{C}(\varepsilon, \varrho)$ belonging to the intensity $\sigma(\varepsilon, \varrho)$ is defined as the expectation value

$$
\boldsymbol{C}(\varepsilon, \varrho)=\boldsymbol{F}(\varepsilon, \varrho)=\overline{\boldsymbol{W}(\varepsilon, \varrho)}-\boldsymbol{D}(\varepsilon, \varrho) .
$$

Under our assumption of vanishing $\boldsymbol{D}$ we get from (1.11) the relation

$$
\boldsymbol{C}(\varepsilon, \varrho)=\bar{M} \boldsymbol{H}^{2}(\varepsilon, \varrho)=\boldsymbol{C}(1,1) \boldsymbol{H}^{2}(\varepsilon, \varrho) .
$$

Inserting this relation into (3.16), we see that in the traces of powers of $\boldsymbol{C}$ we can achieve a diagonalization of $\boldsymbol{C}(\varepsilon, \varrho)$ as well as of $\boldsymbol{C}(1,1)$ and get

$$
\begin{aligned}
& \operatorname{Tr}\left(\boldsymbol{C}^{\varrho}(\varepsilon, \varrho)\right)=\operatorname{Tr}\left(\left(\tilde{\boldsymbol{C}}(1,1) \mathbf{V} \mathbf{H}^{2}(\varepsilon, \varrho) \mathbf{V}^{+}\right)^{\varrho}\right) \\
& \text { or } \quad \sum_{i=1}^{N} \lambda_{i}{ }^{\circ}(\varepsilon, \varrho)=\sum_{i=1}^{N}\left(\sum_{j=1}^{N} \lambda_{j} \eta_{i j}(\varepsilon, \varrho)\right)^{\varrho}
\end{aligned}
$$

where we now understand the $\lambda_{j}$ to be the eigenvalues of $\boldsymbol{C}(1,1)$ and the $\eta_{i j}(\varepsilon, Q)$ the matrix elements of $\boldsymbol{V} \mathbf{H}^{2}(\varepsilon, \varrho) \boldsymbol{V}^{+}$. This last matrix will in general not be diagonal, but it has the property that it transforms a positive vector (having only positive components) into another positive vector. The last expression in (4.6) says that in each particular experiment on the same reaction the eigenvalues of $\tilde{\boldsymbol{C}}(1,1)$ are rotated in a specific way and therefore produce another sequence of effective channel numbers for each experiment. We have not yet found specific relations between these sequences and do not know the extent to which the $\lambda_{j}$ can already fix the range of the other $\lambda_{i}(\varepsilon, Q)$.

We should mention that the same arguments hold, if we start from the representation (1.30) of the reaction intensities, which refers to the reduced $S$-matrix.

A look at Table 2 shows that in the sequence of moments, each next higher order moment contains just one more effective channel number of the corresponding rank. From (3.22) one can infer that this rule is generally true. Therefore the $N_{k}$ can in principle be determined, if the $N$ lowest moments of the intensity were known. The $N_{k}$ in their turn can be considered as the inhomogeneities of a system of algebraic equations for the eigenvalues $\lambda_{i}$ according to (4.3). From this observation we conclude that the $\lambda_{i}$ are in principle observable quantities, though in practice the limited size of the available samples will prohibit this kind of statistical inference.

We will now turn to the question of the extent to which the effective channel numbers may differ from case to case if the eigenvalues $\lambda_{i}$ are allowed to assume arbitrary values. Since we want to utilize some well established mathematical results on expressions like (4.3) we at first bring them into a convenient 
form by introducing the following normalized quantities

$$
x_{i}=\lambda_{i} / \sum_{i=1}^{N} \lambda_{i}
$$

which satisfy the relations

$$
0 \leqq x_{i} \leqq 1
$$

and

$$
\sum x_{i}=1
$$

and allow us to write the effective numbers in the form

$$
N_{k}=\left[\sum_{i=1}^{N} x_{i}^{k}\right]^{-1 /(k-1)}
$$

Quantities of this form are closely related to the so called "means of "rank $t$ " which are defined as a generalization of arithmetic, geometric and harmonic means ${ }^{15}$.

For an array of positive values $x_{i}(i=1, \ldots, N)$ and positive weights $\alpha_{i}>0$ with $\Sigma \alpha_{i}=1$ and any real $t \neq 0$ the mean of rank $t$ is defined by

$$
M_{t}(x, \alpha)=\left[\sum_{i=1}^{N} \alpha_{i} x_{i}^{t}\right]^{1 / t} .
$$

For $t=0$, and $\pm \infty, M_{t}$ can be defined by continuous limits in $t$. As a generalization of the wellknown inequality between the arithmetic and geometric means one can prove that $M_{t}$ is a nondecreasing function of $t$ for $-\infty \leqq t \leqq+\infty$, and that it is strictly increasing unless all the $x_{i}$ are equal.

In a similar way the effective numbers $N_{k}$, formed from the non-negative quantities $x_{i}$, can be considered as a function of any non negative real $k$. According to the definition (4.10) we can say that

$$
N_{k}=\mathbf{l} / M_{k-1}(x, x) \text {. }
$$

In this mean of rank $r=1$, we interpret the $x_{i}$ as their own weights. The restriction to non negative values of $k$ is demanded since in our case some of the eigenvalues $x_{i}$ can accidentally be zero. In order to get a continuous function of $r$, we have to define $N_{k}$ for $k=0,1$ and $+\infty$ by the relations

$$
\begin{aligned}
N_{0} & =N, \\
N_{1} & =\prod_{i=1}^{N} x_{\mathrm{i}}^{-x_{i}}, \\
N_{\infty} & =1 / \max \left(x_{i}\right) .
\end{aligned}
$$

15 E. F. Beckenbach and R. Bellman, Inequalities, SpringerVerlag, Berlin 1961.
Concerning our effective channel numbers we can now say that $N_{k}$ is a non increasing function of $k$, which is strictly decreasing unless all non vanishing $x_{i}$ are equal. From this theorem we immediately derive the following chain of inequalities

$$
N \geqq N_{2} \geqq N_{k} \geqq N_{\infty} \geqq 1
$$

for the effective numbers $N_{k}$ appearing in moments of the intensity $\sigma$. These inequalities restrict the range of the effective numbers in (3.22) quite strongly and irrespectively of the actual value of the correlation matrix $\boldsymbol{C}$ and of the distribution of its eigenvalues $\lambda_{i}$.

If the non vanishing eigenvalues are all equal we have $N_{\infty}=N$. If only one eigenvalue is large and all the others are very small compared to it we get $N_{2} \approx 1$. In both cases the sequence of the effective numbers is independent of the rank $k$. But also in any other case the $N_{k}$ will converge rather quickly against $N_{\infty}$. A general estimate for the least rate of convergence can be derived from Jensen's inequality, which applies to the so called "sums of rank $t$ "

$$
S_{t}=\left[\sum_{i=1}^{N} x_{i}^{t}\right]^{1 / t}
$$

and says that $S_{t}$ as a function of $t$ is non increasing for $0 \leqq t \leqq \infty$. Since we can relate our effective numbers to these sums by

$$
N_{k}=S_{k}^{-k /(k-1)}
$$

we learn that the roots $N_{k}{ }^{(k-1) / k}$ form a non decreasing chain

$$
1 \leqq N_{2}^{1 / 2} \leqq N_{k}^{(k-1) / k} \leqq N_{\infty} .
$$

Combining (4.17) with (4.14) we find that

$$
1 \leqq N_{k} / N_{\infty} \leqq N_{k}^{1 / k} \leqq N_{2}^{1 / k} \leqq N^{1 / k} \text {. }
$$

This relation can serve to estimate the convergence of $N_{k}$ against $N_{\infty}$ more strictly.

The bounds of $N_{k}$ expressed by (4.14), (4.17) and (4.18) guarantee that the sequence of moments (3.22) characterizing the probability distribution of the intensity will not deviate very much from that of a $\chi^{2}$-distribution describing $N_{\text {eff }}$ equally strong reaction channels, provided that an appropriate value for $N_{\text {eff }}$ is chosen between $N_{2}$ and $N_{\infty}$. The choice of $N_{\text {eff }}$ is the less critical the closer the ratio $N_{2} / N_{\infty}$ is equal to one.

From the inequalities (4.14) and from (3.22) one sees that the moments of the probability distribution $P(\varrho)$ of the normalized intensity $\varrho$ are 
bounded by the moments (3.23) of a $\chi^{2}$-distribution of $2 N_{2}$ and $2 N_{\infty}$ degrees of freedom, respectively

$$
g_{r}\left(N_{2}\right) \leqq m_{r}(\varrho) \leqq g_{r}\left(N_{\infty}\right) .
$$

From the inequalities (4.17) on the other side we find that

$$
\frac{1}{N_{k^{k}}-1} \geqq \frac{1}{N_{\infty}^{k}} .
$$

With the help of these relations one can show that the radii of convergence of the TAYLoR expansion for the LAPLACE transform of $P(\varrho)$ and that of a $\chi^{2}$-distribution of $2 N$ d.f. are equal. From this it follows that these two probability densities must asymptotically behave in the same way

$$
P(\varrho) \exp \left\{N_{\infty} \varrho\right\} \underset{\varrho \rightarrow \infty}{\rightarrow} \text { const . }
$$

The present considerations show that $N, N_{2}$, and $N_{\infty}$ are the essential parameters which determine the probability distribution of a normalized intensity as being essentially a $\chi^{2}$-distribution with $2 N_{2}$ d.f. in the vicinity of $\varrho=1$, but having a tail of a $\chi^{2}$-distribution of $2 N_{\infty}$ d. f. The ratio $N_{2} / N_{\infty}$ seems to be the appropriate parameter to measure the deviations of the probability distribution $P(\varrho)$ from a $\chi^{2}$-distribution. For this reason it is interesting to know the maximum value this ratio can have if we allow the eigenvalues $x_{i}$ to have arbitrary values. In order to represent the full variety of possible distributions of the $x_{i}$ we can interpret them as the components of a vector in the $N$-dimensional $x$-space ${ }^{16}$. The end of this vector must point into the positive quadrant of the $x$-space and must lie on the $(N-1)$ dimensional simplex spanned by the $N$ unit vectors in this quadrant because of (4.8) and (4.9). The points of the whole simplex will uniquely represent all possible distributions of the $x_{i}$. In this representation one immediately sees which distribution will maximize the ratio

$$
N_{2} / N_{\infty}=\max \left(x_{i}\right) / \Sigma x_{i}{ }^{2} .
$$

The admissible vectors $x$ which represent distributions with fixed $N_{2}$ lie on a circle which is formed by the intersection of the sphere $\Sigma x_{i}^{2}=1 / N_{2}$ with the simplex $\Sigma x_{i}=1$. Among these vectors all those which lie closest to a vertex of the simplex possess a larger $\max \left(x_{i}\right)$ than all the others. In this way we see from (4.22) that those distributions which possess $(N-1)$ equal eigenvalues and a single larger one will result in a maximum value for $N_{2} / N_{\infty}$.

16 R. S. Ingarden, Fortschr. Physik 13, 755 [1965].
Evaluating the conditions imposed by these considerations we find the extremizing distribution to be characterized by the one eigenvalue accepting the maximum value

$$
\max \left(\lambda_{i}\right)=1 / \sqrt{N}
$$

while the other $(N-1)$ are all equal to

$$
\lambda=1 /(\sqrt{N}(\sqrt{N}+1)) .
$$

The sequence of effective channel numbers belonging to this distribution of eigenvalues can be calculated from the expressions

$$
\begin{aligned}
& N_{k}=\sqrt{N}(\sqrt{N}+1)\left[\frac{\sqrt{N}}{(\sqrt{N}+1)^{k-1}+\sqrt{N}-1}\right]^{1 /(k-1)}, \\
& N_{\infty}=\sqrt{N} .
\end{aligned}
$$

The corresponding maximum value of $N_{2} / N_{\infty}$ is given by

$$
N_{2} / N_{\infty}=(\sqrt{N}+1) / 2 .
$$

In general a given value of $N_{2}$ besides the well known $N$ will restrict the possible values of $N_{\infty}$ and thereby the values of the other $N_{k}$ more than in this extreme case. From similar arguments as those applied above one finds that the following bound for $N_{\infty}$ can be given

$N_{2} \geqq N_{\infty} \geqq N /\left(1+\sqrt{\left(N-N_{2}\right)(N-1) / N_{2}}\right)$.

Though there is no reason to expect just the distribution $(4.23),(4.24)$ of the $\lambda_{i}$ to be realized in any nuclear reaction, we think that the corresponding probability density $P_{\mathrm{m}}(\varrho)$ can illustrate a case of extreme deviations from a pure $\chi^{2}$-distribution. The density $P_{\mathrm{m}}(\varrho)$ can be calculated as the density of the sum of two random numbers, one distributed with a $\chi^{2}$-distribution of $2 \mathrm{~d}$.f. and a mean value (4.23), the other one with a $\chi^{2}$-distribution of $2(N-1)$ d. f. and the mean $(\sqrt{N}-1) / \sqrt{N}$ according to $(4.24)$. The result of this calculation is given by

$$
\begin{aligned}
P_{\mathrm{m}}(\varrho) \mathrm{d} \varrho= & \left(\frac{N-1}{\sqrt{N}-1}\right)^{N-1} N^{1-(N / 2)} e^{-v \bar{N} \varrho} \\
& \times\left[1-e^{-N} \varrho \sum_{\nu=1}^{N-2} \frac{(N \varrho)^{\nu}}{\nu !}\right] \mathrm{d} \varrho .
\end{aligned}
$$

In Fig. 1 we have depicted this density for the particular case where $N=25, N_{2}=15, N_{\infty}=5$. Compared with a $\chi^{2}$-distribution of $2 \mathrm{~N}_{2}$ d.f. the density $P_{\mathrm{m}}$ is a little more slender and its tail drops down less rapidly than that of the corresponding $\chi^{2}$-distribution of $2 N_{2}$ d.f., as we were led to expect by 


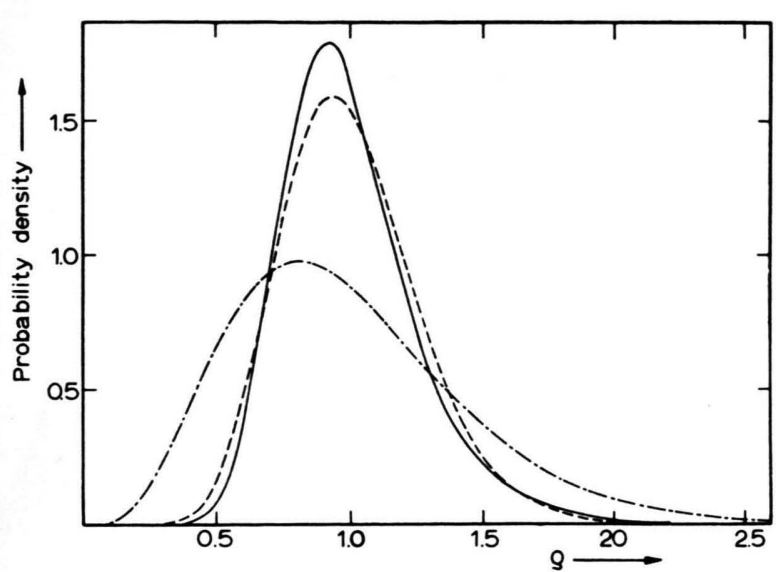

Fig. 1. Plot of the probability distribution $P_{\mathrm{m}}(\varrho)$ and of $\chi^{2}$-distributions. --15 d.f.; $\cdots .5$ d.f.; $\longrightarrow P_{\mathrm{m}}(\varrho)$.

(4.21). But this $\chi^{2}$-distribution of $2 N_{\infty}$ d.f. on the other side has very little resemblance with $P_{\mathrm{m}}$ for small values of $\varrho$.
We should mention that the expansion of the density $P_{\mathrm{m}}(\varrho)$ into an EDGeworth' series turned out to become unstable from the second term on. The approximation of the probability density for any intensity by a few terms of the expansion

$P(\varrho)=\frac{N_{2}}{\Gamma\left(N_{2}\right)}\left(\sum_{n=1}^{\infty} a_{n} \varrho^{n}\right) \varrho^{N_{2}-1} \exp \left\{-N_{\infty} \varrho\right\}$

which can be accomplished by adjusting the first few moments works much better.

\section{Acknowledgement}

I wish to thank Professor W. Gentner for the generous support of this work and the kind hospitality which I enjoyed in his institute. I am indebted to Prof. H. A. Weidenmüller, Dr. C. Noack and A. Richter and thank them for valuable discussions and their interest in this work. 